\title{
Massive MIMO Adaptive Modulation and Coding Using Online Deep Learning
}

\author{
Evgeny Bobrov, Dmitry Kropotov, Hao Lu, Danila Zaev
}

\begin{abstract}
The paper describes an online deep learning algorithm for the adaptive modulation and coding in 5G Massive MIMO. The algorithm is based on a fully connected neural network, which is initially trained on the output of the traditional algorithm and then is incrementally retrained by the service feedback of its output. We show the advantage of our solution over the state-ofthe-art Q-Learning approach. We provide system-level simulation results to support this conclusion in various scenarios with different channel characteristics and different user speeds. Compared with traditional OLLA our algorithm shows $10 \%$ to $20 \%$ improvement of user throughput in full buffer case.
\end{abstract}

Index Terms-adaptive modulation and coding, link adaptation, olla, deep learning, reinforcement learning, massive MIMO, wireless communications, online training.

\section{INTRODUCTION}

The adaptive modulation and coding (AMC) process carried out in the link adaptation is a crucial part of current wireless communication systems. It becomes especially important and challenging in Massive MIMO systems with dynamic beamforming. Advanced AMC techniques allow to significantly increase the data rate that can be reliably transmitted [1].

In the downlink AMC procedure [2], the user equipment (UE) has to suggest to the base station (BS) an appropriate modulation and coding scheme (MCS) to be used in the next transmission. The proposed MCS is signaled from the UE using a channel quality indicator (CQI). Each CQI is associated with a particular signal-to-inference-and-noise ratio (SINR) interval. In Massive MIMO systems, the accuracy of CQI is limited by the number of CSI-RS antenna ports, which is usually less than the number of transmit antennas at BS. In TDD systems SRSbased channel knowledge can be utilized to compensate for the CQI inaccuracy, but the actual SINR is still difficult to estimate due to the difference in the receiver algorithms quality.

The well-known outer loop link adaptation (OLLA) technique was first proposed in [3]. OLLA modifies the SINR CQI-based estimation by an offset $[4,5]$ which can be positive (making the MCS selection more conservative) or negative (when the CQI selection was too optimistic). This offset is updated based on transport blocks' transmission success rate so that the average block error rate is kept as close as possible to the predefined target [6].

The work was supported by Huawei Technologies. Evgeny Bobrov is with Moscow Research Center, Huawei Technologies, Russia, and M. V. Lomonosov Moscow State University, Russia (e-mail: eugenbobrov@ya.ru). Dmitry Kropotov is with National Research University Higher School of Economics, Russia, and M. V. Lomonosov Moscow State University, Russia (e-mail: dkropotov@yandex.ru). Hao Lu and Danila Zaev are with Moscow Research Center, Huawei Technologies, Russia (e-mail: luhao12@ huawei.com, zaev.danila1@huawei.com).
OLLA family of algorithms use only the last binary acknowledgment information and does not take into account more refined SINR channel data, available e.g. via sounding (SRS) measurements. We offer an adaptive and self-learning method that predicts the next MCS using the current SINR. In other words, it performs the mapping from SINR channel data to the optimal MCS, and training occurs in an online manner.

The main advantage of the proposed online deep learning (ODL) algorithm is that it can be adapted to any environment with any conditions, which BS is not able to measure directly, such as the agent speed. Different agent speed at the same SINR has a different meaning and even if we use the pre-trained offline algorithm on various collected examples of speeds, we couldn't distinguish between them on the test. In the literature, this situation is called Concept Drift [7] - when some hidden features are important, but cannot be measured and change over time. This way our task falls into the class of Incremental Learning [8] algorithms which proceed with optimization in non-stationary environments such as the Massive MIMO system we are working on. The deep learning approach in MIMO also is studied in the work [9].

In our work, we show that the ODL based algorithm can adapt to the new environment and that is why significantly outperform the traditional OLLA solution and the more sophisticated Q-learning approach. The computational complexity and storage requirements of the ODL approach have been investigated. From this study, we have got stable results that uniformly outperform the baseline. The proposal increases throughput value on average from $10 \%$ to $20 \%$ compared to OLLA depending on the agent speed.

This paper is organized as follows. Section 2 briefly describes the massive MIMO model. Section 3 carries out the proposed algorithm structure, the neural network model, and its complexity in online training using the sample buffer approach. Section 4 describes the main experimental results. Section 5 contains the conclusion.

\section{SYSTEM MODEL}

In the MIMO system, it is possible to send several information symbols to the same multi-antenna user simultaneously at the same sub-band. The number of such symbols is called the rank of the user. Under certain channel conditions the greater rank can significantly increase the amount of transmitted information, but at the same time increases requirements for channel reliability. The Single-User MIMO model is described by the following linear system:

$$
r=G(H W x+n)
$$


Where $r \in C^{L}$ is a vector of detected symbols at receiver, $x \in$ $C^{L}$ is a vector of sent symbols, $H \in C^{R \times T}$ is a channel matrix, $W \in C^{T \times L}$ is a precoding matrix, $G \in C^{L \times R}$ is a detection matrix, and $n \sim C N\left(0, I_{L}\right)$ is a noise-vector. The constant $T$ is the number of transmit antennas, $R$ is the number of transmit antennas, $L$ is the user rank. We assume they are related as $L \leq$ $R \leq T$. As a detection matrix $G$ we use MMSE [13] and for the $W$ precoding rank reduction, we use SVD [14].

The common optimization objective is to maximize Spectral Efficiency (SE) of MCS multiplied by the probability of successful transmission (ACK), which is also called Throughput [15]:

$$
p(a c k \mid m c s, \sin r) S E(m c s) \rightarrow \max _{m c s}
$$

\section{STRUCTURE OF THE PROPOSED ALGORITHMS}

The general structure of the solution follows [6] since the algorithm predicts success acknowledgment (ACK) probability for each MCS, given measured SINR values. We propose to multiply the probability of success by the Spectral Efficiency for each MCS and take the maximum argument:

$$
\widehat{m c S_{S E}}(\sin r)=\underset{m c s}{\arg \max }\left\{p_{w}(a c k \mid m c s, \sin r) S E(m c s)\right\}
$$

Here $p_{w}(a c k \mid m c s, \sin r)$ is a neural network model which predicts probabilities and has weights " $w$ " as the parameters for optimization. The neural network takes as input the frequencyspecific SINR estimations and iterates through the MCS values. A proper $p_{w}$ model is a decreasing function of MCS. Therefore, it should be reweighted with some increasing function of MCS: $S E(m c s)$, but other approaches to define the value are also possible. The algorithm suggests applying the MCS with the maximum expected value.

In the recent state-of-the-arts, there is a tendency to use the Reinforcement Learning (Q-learning) technique for choosing the proper MCS like an agent actions [10,11]. Despite the deep Q-learning network (DQN) is widely used in wireless communication systems and can work in this task, we offer the scheme (3) using classical deep learning that is superior to DQN. Key ideas of the MCS problem:

a) All actions are performed immediately and there are no delayed rewards, like in a chess game, that is modeled by DQN. b) There is no influence on the system by our actions. The following SINRs are independent of the MCS we choose.

c) The system works dynamically. We have access to the following features and labels sequentially.

Assumptions $a$ ) and $b$ ) lead us to try the classical deep learning approach, considering the binary classification problem and the scheme (2) of choosing the optimal MCS. Assumption $c$ ) forces us to use the online approach. Let us analyze these points.

Under the assumptions of dynamic interaction with the system, our approach works on similar principles to DQN. The main difference is that we use Binary Log-Loss instead of Q-learning TD-Loss [12]. In such a way, we move to the binary classification problem instead of maximizing the delayed rewards $b$ ) and modeling the influence on the system by our actions $a$ ).

We insist that TD-Loss is excessive since we do not affect the system with our MCS selection and do not need to learn the agent behavior, i.e. the chain of the future actions, which we usually meet in chess and other games for which ODL is applied. Based on this motivation, we suggest selecting MCS only for the next step, which makes the algorithm computationally simpler, makes it more appropriate to the MCS problem, and, at the same time, and improves quality.

Fig 1. Online Deep Learning algorithm block scheme.

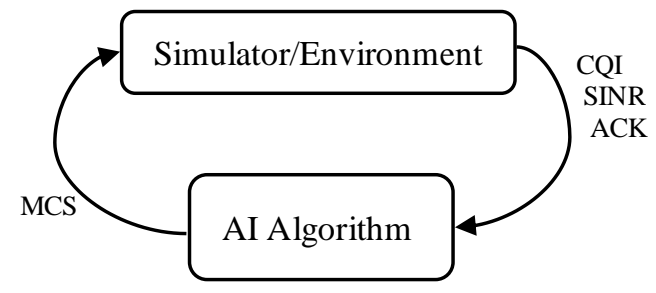

As a competitor of our solution we consider the following QLearning regression model $[10,11]$, which chooses MCS by taking the maximum argument:

$$
\widehat{m c s_{S E}}(\sin r)=\underset{m c s}{\arg \max }\left\{q_{w}(\operatorname{ack} \mid m c s, \sin r)\right\}
$$

Here $q_{w}(a c k \mid m c s, \sin r)$ is the neural network regression, which predicts real scalar values. The Q-Learning model is trained on the rewards $r(a c k, m c s)=S E(m c s)[a c k]$, where $[x]$ is the indicator function, which takes a value 1 , if condition $x$ is true, and value 0 otherwise. The condition ack corresponds to the receipt of the success acknowledgment. We will discuss this in more detail in the next section.

\section{B. Neural Network Model}

In that work, we are using a simple neural network for binary classification with two hidden layers. And so, the model is lightweight, fast trainable, and robust to the environmental changes in the online-learning setting.

Our classification model uses the standard sigmoid function, which takes any real input $t$, and outputs a value between zero and one. The sigmoid function $\sigma: \mathbb{R} \rightarrow(0,1)$ is defined as follows: $(t)=\frac{1}{1+e^{-t}}$.

Thus, we can express the probability of receiving acknowledge in terms of sigmoid function $\sigma$ depending on $m c s$ and sinr arguments, where $f_{w}$ is the neural network function with weights $w$ :

$$
p_{w}(a c k \mid m c s, \sin r)=\sigma\left(f_{w}(m c s, \sin r)\right)=\frac{1}{1+e^{-f_{w}(m c s, \sin r)}}
$$

The output of the model for a given observation, given a vector of input features, can be interpreted as a probability, which serves as the basis for classification. The optimization method computes the log-loss for all the observations $n \in 1 \ldots N$ on which it is trained. The function $J$ counts the log-probabilities of ACKs in the following way: 
$J(w)=\frac{1}{N} \sum_{n=1}^{N}\left(a c k_{n} \log p_{w}\left(a c k_{n} \mid \operatorname{sinr}_{n}, m c s_{n}\right)+(1-\right.$

$\left.\left.\operatorname{ack}_{n}\right) \log \left(1-p_{w}\left(a c k_{n} \mid \operatorname{sinr}_{n}, m c s_{n}\right)\right)\right) \rightarrow \max _{w}$

Where $a c k_{n} \in\{0,1\}$ is the "true" acknowledge, which we get to know after the action had done and $p_{w}\left(a c k_{n} \mid \sin _{n}, m c s_{n}\right)$ is the modeled probability of the $a c k_{n}$ reception as a function of features: $\left\{\sin _{n}, m c s_{n}\right\}$.

For the Q-Learning approach, we use the MSE-Loss functional on the reward. Since we do not have a delayed reward, it is TDLoss with $\gamma=0$ [12]:

$$
L(w)=\frac{1}{N} \sum_{n=1}^{N}\left(q_{w}\left(a c k_{n} \mid \operatorname{sinr}_{n}, m c s_{n}\right)-r\left(a c k_{n}, m c s_{n}\right)\right)^{2} \rightarrow
$$

\section{Proposed algorithm complexity}

We use Adam as one of the simplest gradient-based algorithms. It can be noticed that we can reuse the previous solution $w_{t}^{*}$ (optimal weights of the model) as the starting point for the next retraining step $w_{t+1}^{o}$, thus: $w_{t+1}^{o}=w_{t}^{*}(8)$. Therefore, it is enough to make just a few gradient steps for such correction.

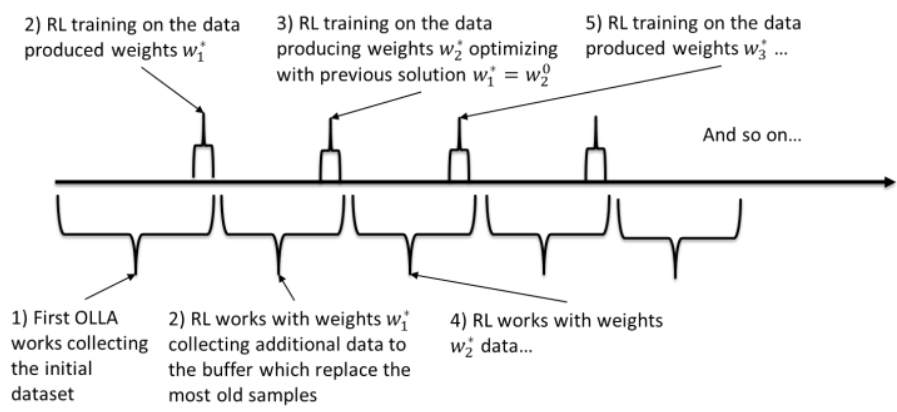

Figure 2. Working Algorithm Time Axis.

Since the algorithm works online, it needs to be retrained on the new data. The current online deep learning model stores a feature memory buffer for every user. It updates samples in the FIFO order, replacing the oldest samples with the newest ones. We can visualize this mechanism using the following scheme of the sample buffer.

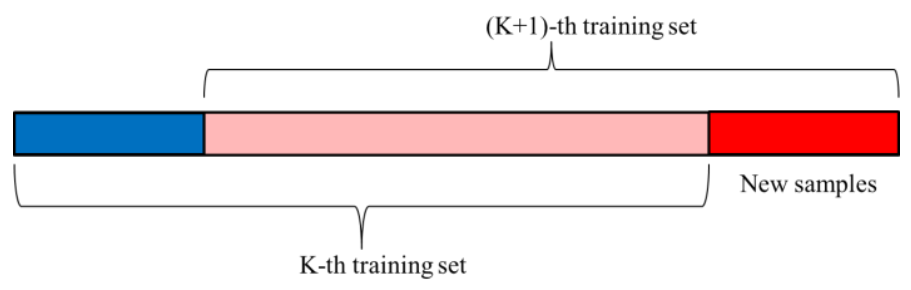

Figure 3. Algorithm Sample Buffer.

We suggest adding new samples to the buffer with a possibly adaptive subsampling rate to avoid the situation, where most features remain the same between the channel measurements. This way we significantly decrease the memory buffer size and the speed of retraining without reducing the quality. The quality may even get better since we can expand the buffer to our storage limits. For our experiments, we chose subsampling rate as an inverse probability of sounding length excluding the pilot signals in the full buffer user conditions.
Table 1. Online Deep Learning algorithm with Sample Buffer.

\begin{tabular}{|l|}
\hline Online Deep Learning with Sample Buffer \\
\hline Parameters: Initial value and step size of OLLA: $y_{o}, u$. \\
Initial CQI: $c_{o}$, target BLER $b$, buffer size: $L$ and retraining period: $R$ \\
\hline Initialize: OLLA: $y=y_{o}$, CQI: $c=c_{o}$. \\
Sample buffer $D$ of the size $L$ and NN model $A(w)$ \\
\hline For 1 to $L$ do \\
\hline Set MCS $\leftarrow$ clip(round $(c+y), 1, k)$ \\
\hline Receive and collect to the buffer $D$ \\
labels: ACK or NACK: $a \in\{0,1\}$, and features: \\
CQI: $c \in\{1 \ldots n\}$, SINR $s \in \mathbb{R}^{m}$, MCS $\in\{1 \ldots k\}$ \\
\hline Update OLLA state: $y \leftarrow y+a u-(1-a) u(1-b) / b[6]$ \\
\hline Training of the NN: $A(w)$ on function $J(6)$ or $L(7)$ and buffer $D$ \\
\hline For each TTI $K$ do \\
\hline Set MCS $\leftarrow$ NN model $A(w)$ prediction by formula (3) or (4) \\
\hline Receive and replace the oldest values of the buffer $D$ \\
labels: ACK or NACK: $a \in\{0,1\}$, and features: \\
CQI: $c \in\{1 \ldots n\}$, SINR $s \in \mathbb{R}^{m}$, MCS $\in\{1 \ldots k\}$ \\
\hline If $K$ mod $R=0$ then \\
\hline Initialize NN weights from the previous training as in (8) \\
After-train NN model $A$ using function $(6)$ or $(7)$ and buffer $D$ \\
\hline End \\
\hline
\end{tabular}

\section{SYSTEM-LEVEL ONLINE SIMULATION RESULTS}

We are comparing our machine learning algorithm mainly with the deterministic OLLA. Therefore, all gains and losses are calculated in comparison with OLLA. We have got stable, uniformly better results, which have never failed in our experiments. It has increased throughput value on average from $12.64 \%$ to $21.52 \%$ depending on speed.

The advantage of the proposal is explained by the usage of additional information like user SINR and layers eigenvalues. We can also notice that the step-by-step behavior of OLLA is too conservative in a rapidly changing environment.

\section{A. Quality improvement with machine learning}

We provide experimental results for different speeds, user ranks, and random seeds. The proposed algorithm is not manually tuned under various conditions, all its hyperparameters remain the same. It is important since in the real-life commercial system BS does not have information about the user speed and, especially, about the user environment.

Table 2. Channel throughput (1e9) and quality gains of the ODL and OLLA algorithms, averaged by 10 random seeds. Ranks 1, 2, 3 .

\begin{tabular}{|l|l|l|l|}
\hline & \multicolumn{3}{|l|}{ Rank 1 } \\
\hline User Speed & OLLA & ODL & Gain \\
\hline $3 \mathrm{~km} / \mathrm{h}$ & 1.71 & 1.96 & $\mathbf{1 4 . 7 6 \%}$ \\
\hline $24 \mathrm{~km} / \mathrm{h}$ & 1.02 & 1.07 & $\mathbf{6 . 1 \%}$ \\
\hline $60 \mathrm{~km} / \mathrm{h}$ & 0.84 & 0.91 & $\mathbf{1 4 . 2 7 \%}$ \\
\hline & Rank 2 \\
\hline User Speed & OLLA & ODL & Gain \\
\hline $3 \mathrm{~km} / \mathrm{h}$ & 2.4 & 2.83 & $\mathbf{1 8 . 1 5 \%}$ \\
\hline $24 \mathrm{~km} / \mathrm{h}$ & 1.28 & 1.38 & $\mathbf{1 0 . 2 3 \%}$ \\
\hline $60 \mathrm{~km} / \mathrm{h}$ & 1.08 & 1.21 & $\mathbf{1 9 . 2 4 \%}$ \\
\hline & Rank 3 & \multicolumn{3}{|l|}{} \\
\hline User speed & OLLA & ODL & Gain \\
\hline $3 \mathrm{~km} / \mathrm{h}$ & 2.4 & 2.83 & $\mathbf{1 8 . 1 5 \%}$ \\
\hline $24 \mathrm{~km} / \mathrm{h}$ & 1.28 & 1.38 & $\mathbf{1 0 . 2 3 \%}$ \\
\hline $60 \mathrm{~km} / \mathrm{h}$ & 1.08 & 1.21 & $\mathbf{1 9 . 2 4 \%}$ \\
\hline
\end{tabular}


The online deep learning model performs the mapping from the SINR feature to the optimal MCS. The main advantage we get on the rises and falls because ODL is much more adaptive to the current SINR than OLLA and instantly converges to the optimal MCS. The following picture shows uniformly the advantage of the ODL algorithm over OLLA.

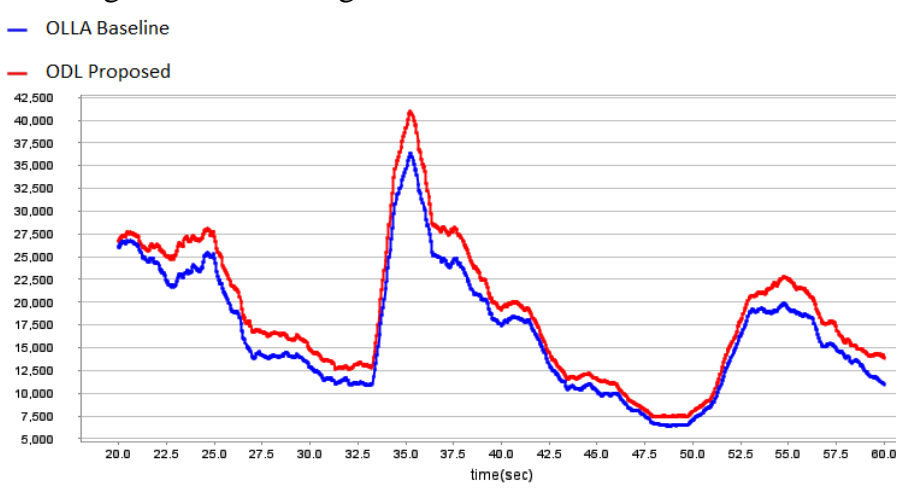

Figure 4. Throughput statistics, second rank, $24 \mathrm{~km} / \mathrm{h}$ speed. The red line is ODL, the blue is OLLA. Moving average with a window of 2 seconds.

\section{B. Probabilistic approach and Q-Learning comparison}

To check our assumptions about the system, we have compared the ODL approach with the Q-Learning one. We show that the ODL method works uniformly better for all ranks at agent speed of $30 \mathrm{~km} / \mathrm{h}$ and random trajectory. Both models use the same neural network architecture and differ only by the activation and loss functions. The ODL method uses ReLu at the inner layers and the sigmoid at the last layer to predict probabilities. The Q-Learning model uses Hyperbolic Tangent at the middle layers and the Identity at the last layer to predict real Q-values.

Table 3. Gain over OLLA of two models: ODL and Q-Learning. The agent speed is $30 \mathrm{~km} / \mathrm{h}$ with a random trajectory for moving.

\begin{tabular}{|l|l|l|l|l|}
\hline & Rank 1 & Rank 2 & Rank 3 & Rank 4 \\
\hline ODL & $\mathbf{8 . 4 7 \%}$ & $\mathbf{1 4 . 3 \%}$ & $\mathbf{2 3 . 7 \%}$ & $\mathbf{2 2 \%}$ \\
\hline Q-Learning & $2.73 \%$ & $\mathbf{7 \%}$ & $16 \%$ & $18 \%$ \\
\hline
\end{tabular}

Table 4. System configuration used in our experiments.

\begin{tabular}{|l|l|}
\hline CellMaxPower & $40 \mathrm{dBm}$ \\
\hline ThermalNoisePower & $-174 \mathrm{dBm} / \mathrm{Hz}$ \\
\hline Bandwith & $20 \mathrm{MHz}$ \\
\hline TxAntNum, $T$ & 64 \\
\hline RxAntNum, $R$ & 4 \\
\hline Sounding Period & $5 \mathrm{~ms}$ \\
\hline
\end{tabular}

V. CONCLUSION

The online deep learning algorithm is a promising solution for the adaptive modulation and coding problem in Massive MIMO systems. It shows stable performance and uniformly better quality compared with both the traditional OLLA and the Qlearning-based neural network solutions. The results are supported by the system-level simulation experiments.

\section{REFERENCES}

[1] Chung, Seong Taek, and Andrea J. Goldsmith. "Degrees of freedom in adaptive modulation: a unified view." IEEE Transactions on Communications 49.9 (2001): 1561-1571

[2] 3GPP TS 36.211, Evolved universal terrestrial radio access (E-UTRA); Physical Channels and Modulation (Release 10), V10.7.0, (Sophia Antipolis Valbonne, France, 2013)

[3] A Sampath, P Sarath Kumar, JM Holtzman, in IEEE Vehicular Technology Conference, vol. 2. On setting reverse link target SIR in a CDMA system, (1997), pp. 929-9332.

[4] P Song, S Jin, in International Conference on Communications and Information Technology (ICCIT). Performance evaluation on dynamic dual layer beamforming transmission in TDD LTE system, (2013), pp. 269-274

[5] KI Pedersen, G Monghal, IZ Kovacs, TE Kolding, A Pokhariyal, F Frederiksen, et al, in IEEE Vehicular Technology Conference (VTC Fall), Baltimore, USA. Frequency domain scheduling for OFDMA with limited and noisy channel feedback, (2007), pp. 1792-1796

[6] Blanquez-Casado, Francisco, et al. "eOLLA: an enhanced outer loop link adaptation for cellular networks." EURASIP Journal on Wireless Communications and Networking 2016.1 (2016): 20.

[7] Gama, João, et al. "A survey on concept drift adaptation." ACM computing surveys (CSUR) 46.4 (2014): 1-37.

[8] Krawczyk, Bartosz, and Alberto Cano. "Online ensemble learning with abstaining classifiers for drifting and noisy data streams." Applied Soft Computing 68 (2018): 677-692.

[9] Wen, Chao-Kai, Wan-Ting Shih, and Shi Jin. "Deep learning for massive MIMO CSI feedback." IEEE Wireless Communications Letters 7.5 (2018): 748-751.

[10] Mota, Mateus P., et al. "Adaptive Modulation and Coding based on Reinforcement Learning for 5G Networks." 2019 IEEE Globecom Workshops (GC Wkshps). IEEE, 2019.

[11] Zhang, Lin, et al. "Deep reinforcement learning-based modulation and coding scheme selection in cognitive heterogeneous networks." IEEE Transactions on Wireless Communications 18.6 (2019): 3281-3294.

[12] Tesauro, Gerald. "Temporal difference learning and TD-Gammon." Communications of the ACM 38.3 (1995): 58-68.

[13] Wubben, D., Bohnke, R., Kuhn, V., I\& Kammeyer, K. D. (2004, June). Near-maximum-likelihood detection of MIMO systems using MMSEbased lattice-reduction. In 2004 IEEE International Conference on Communications (IEEE Cat. No. 04CH37577) (Vol. 2, pp. 798-802). IEEE.

[14] Sun, Liang, and Matthew R. McKay. "Eigen-based transceivers for the MIMO broadcast channel with semi-orthogonal user selection." IEEE Transactions on Signal Processing 58.10 (2010): 5246-5261

[15] Fan, Pingyi, and Khaled Ben Letaief. "Understanding of transmission throughput and channel capacity in a systematic way." 2011 20th Annual Wireless and Optical Communications Conference (WOCC). IEEE, 2011.

\section{APPENDIX}

An agent trajectory draws like Brownian movement on the plane. The trajectory of a single random seed remains the same at different distances and speeds. In some seeds, the agent keeps its distance from the station and sometimes even moves through it. At low speed, the agent follows a much smaller trajectory, although it follows the same path.
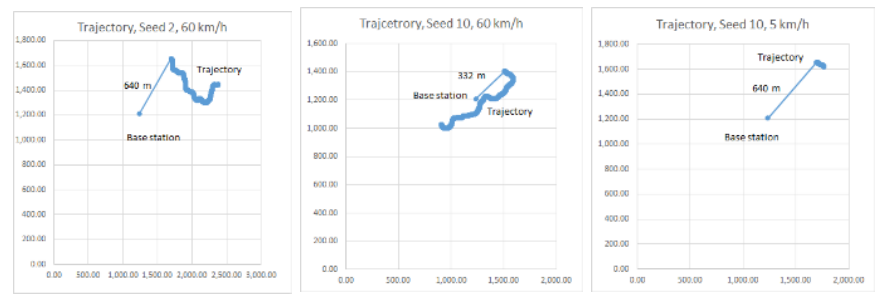

Figure 5. Example of the agent trajectories. 